\title{
Vortex Condensation in the Chern-Simons Higgs Model: An Existence Theorem
}

\author{
Luis A. Caffarelli, Yisong Yang ${ }^{\star}$ \\ School of Mathematics, Institute for Advanced Study, Princeton, NJ 08540, USA
}

Received: 8 December 1993

\begin{abstract}
It is shown that there is a critical value of the Chern-Simons coupling parameter so that, below the value, there exists self-dual doubly periodic vortex solutions, and, above the value, the vortices are absent. Solutions of such a nature indicate the existence of dyon condensates carrying quantized electric and magnetic charges.
\end{abstract}

\section{Introduction}

In this paper, we shall concentrate on a mathematically simplified anyon model known as the Abelian Chern-Simons Higgs model in which the Yang-Mills or the Maxwell field term is dropped and the Higgs potential takes a specific form. The dominance of the Chern-Simons gauge field gives rise to both electrically and magnetically charged vortices known as dyons. In the recent work [HKP, JW], it is found that there exists a self-dual structure so that the model permits a class of topological multivortex solutions with quantized charges similar to the solutions in the Abelian Higgs equations [JT, T1, T2] and a class of nontopological solutions carrying fractional values of charges [CHMcY, JLW, SY1]. This raises hope to establish the existence of condensates or periodic multivortices in the model. In this paper, we will present such a result. Note that the vortex condensation phenomenon in a gauged nonlinear Schrödinger equation has been established in [O]. There some evidence, which is consistent with our result here, is also given that there may exist periodic multivortices in the full self-dual Chern-Simons Higgs model when the basic lattice cell is sufficiently large so that its approximation by the gauged Schrödinger equation becomes effective.

In our problem, the self-duality condition requires the specific assumption that the Higgs potential be of a $\phi^{6}$ type which limits, of course, the applicability of the theory to general situations. On the other hand, however, the mathematics here is certainly richer than the classical self-dual Abelian Higgs model where the potential \footnotetext{
USA

Present address: Department of Mathematics, Polytechnic University, Brooklyn, NY 11201,
} 
function is of a $\phi^{4}$ type. In the Chern-Simons model the topological solutions [SY2] asymptotically recover the solutions in the Abelian Higgs model. Besides, nontopological solutions, which are absent in the Abelian Higgs case, are present in the Chern-Simons case and give rise to fractional values of electric and magnetic charges and a continuous spectrum of energies.

In its full setting, the equations of motion of the anyon model assumes a difficult form. However, it may be useful to mention that there has been considerable attention on the model recently (see [FM1, FM2] and references therein). Mathematically, the anyon model is a classical field theory defined on the $(2+1)$-dimensional Minkowski space $\mathbf{R}^{2,1}$ and contains the coupling of the scalar fields and the YangMills and Chern-Simons gauge fields. The condensate solutions have been discussed by many people (see [CH, H, IL, P, RSS]) and it is believed that these solutions are relevant to various important issues in theoretical physics such as high-temperature superconductivity and the quantum Hall effect. Therefore it is of interest to pursue a mathematical study of the existence of the condensates or stationary periodic vortices in the anyon model, or sometimes, its simplifications such as the one studied here.

An interesting connection may be made of our problem with Abrikosov's mixed states $[\mathrm{Ab}]$ in a type-II superconductor, also known as condensates, whose existence is due to a sublevel magnetic excitation. There the excited state always exhibits a lattice type periodic structure so that the total magnetic flux through a basic cell can only assume a few selected values related to the local defects. These defects are represented by the zeros of the order parameter and are indications of a partial destruction of superconductivity. The order parameter is just the Higgs scalar and the quantized flux is characterized by the winding number of the scalar field around the boundary of a lattice cell. In fact, the winding number is actually related to the number of algebraic zeros of the field confined in the interior of the cell. It is already known that, in the self-dual coupling, such a picture may be realized exactly and the number of zeros of the Higgs field or the vortex number for a solution in a periodic region is bounded from above by a threshold depending on the size of the region [WY], but independent of the locations of the vortices. Our analysis in the Chern-Simons case here seems to indicate that a similar threshold depends on these locations.

Here is an outline of the contents of the paper to follow. In the next section, we derive the governing self-dual equations for the Chern-Simons vortices in a periodic region using the method of $[\mathrm{JW}]$ and the 't Hooft periodic boundary condition ['t H]. We then state our existence theorem. In Sect.3, we use a sub/super solution technique to construct the solutions of the underlying elliptic equation. In Sect.4, we sketch an alternative variational approach to the problem.

\section{Periodic Multivortices}

We adapt the notation in [JW]. The $(2+1)$-dimensional Minkowski spacetime metric is $\operatorname{diag}(1,-1,-1)$ which will be used to raise or lower indices. The Lagrangian action density of the Chern-Simons Higgs theory is

$$
\mathscr{L}=\left(D_{\mu} \phi\right)\left(D^{\mu} \phi\right)^{*}+\frac{1}{4} \kappa \varepsilon^{\alpha \beta \gamma} A_{\alpha} F_{\beta \gamma}-V(|\phi|),
$$

where $D_{\mu}=\partial_{\mu}-\mathrm{i} A_{\mu}, A_{\mu}$ is a 3-vector field called the Chern-Simons gauge field, $\phi$ a complex scalar field called the Higgs field, $F_{\alpha \beta}=\partial_{\alpha} A_{\beta}-\partial_{\beta} A_{\alpha}, \alpha, \beta, \gamma, \mu=$ 
$0,1,2, \kappa>0$ the coupling parameter, $\varepsilon^{\alpha \beta \gamma}$ totally skew-symmetric with $\varepsilon^{012}=1$, and $V$ the Higgs potential function.

The Euler-Lagrange equations associated with the action density $\mathscr{L}$ are

$$
\begin{aligned}
\frac{1}{2} \kappa \varepsilon^{\alpha \beta \gamma} F_{\beta \gamma} & =j^{\alpha}=\mathrm{i}\left(\phi\left[D^{\alpha} \phi\right]^{*}-\phi^{*}\left[D^{\alpha} \phi\right]\right), \\
D_{\mu} D^{\mu} \phi & =-\frac{\partial V}{\partial \phi^{*}},
\end{aligned}
$$

where $j^{\alpha}=(\rho, \mathbf{j})$ is the conserved matter current density and $F_{12}$ the magnetic field. In the self-dual model [HKP, JW], the potential function takes the triple-well form

$$
V(|\phi|)=\frac{1}{\kappa^{2}}|\phi|^{2}\left(1-|\phi|^{2}\right)^{2} .
$$

In the rest of the paper, we shall always observe this assumption.

We are to look for stationary solutions of the Chern-Simons equations (1) over a periodic cell with a gauge-periodic boundary condition to be specified later. Since the $\alpha=0$ component of the first equation in (1) reads

$$
\kappa F_{12}=j^{0}=\rho=-2 A_{0}|\phi|^{2},
$$

the magnetic flux $\Phi$ and the electric charge $Q$ are related by the equation

$$
\kappa \Phi=\kappa \int F_{12} d x=\int \rho d x=Q .
$$

On the other hand, it is straightforward to see that the energy-momentum tensor of $\mathscr{L}$ is given by

$$
T_{\mu v^{\prime}}=2 \operatorname{Re}\left\{\left(D_{\mu} \phi\right)^{*}\left(D_{v} \phi\right)\right\}-g_{\mu \nu}\left[\left(D_{\gamma} \phi\right)\left(D^{\gamma} \phi\right)^{*}-V(|\phi|)\right] .
$$

Thus the energy density $\mathscr{E}=T_{00}$ is written in view of (2) in the form

$$
\mathscr{E}=\frac{\kappa^{2}}{4} \cdot \frac{F_{12}^{2}}{|\phi|^{2}}+\left|D_{j} \phi\right|^{2}+\frac{1}{\kappa^{2}}|\phi|^{2}\left(1-|\phi|^{2}\right)^{2},
$$

where $j=1,2$.

In order to introduce a suitable periodic boundary condition, we recall the gaugesymmetry of $\mathscr{L}$ given by the general expressions

$$
\phi \mapsto \phi \mathrm{e}^{i \omega}, \quad A_{\mu} \mapsto A_{\mu}+\partial_{\mu} \omega,
$$

Since we are interested in stationary field configurations, the above gauge-symmetry becomes

$$
\phi \mapsto \phi \mathrm{e}^{\mathrm{i} \omega}, \quad A_{0} \mapsto A_{0}, \quad A_{j} \mapsto A_{j}+\partial_{j} \omega,
$$

where $\omega$ is a real-valued function of the spatial coordinates $x_{j}(j=1,2)$ only.

We are now ready to examine the 't Hooft boundary condition.

Consider a basic lattice cell $\Omega$ in $\mathbf{R}^{2}$ generated by independent vectors $a^{1}$ and $a^{2}$ :

$$
\Omega=\left\{x=\left(x_{1}, x_{2}\right) \in \mathbf{R}^{2} \mid x=s_{1} a^{1}+s_{2} a^{2}, 0<s_{1}, s_{2}<1\right\} .
$$

Define

$$
\Gamma^{k}=\left\{x \in \mathbf{R}^{2} \mid x=s_{k} a^{k}, 0<s_{k}<1\right\}, \quad k=1,2 .
$$

Then the boundary of $\Omega$ is given by

$$
\partial \Omega=\Gamma^{1} \cup \Gamma^{2} \cup\left\{a^{1}+\Gamma^{2}\right\} \cup\left\{a^{2}+\Gamma^{1}\right\} \cup\left\{0, a^{1}, a^{2}, a^{1}+a^{2}\right\} .
$$


In view of the gauge transformation (5), we impose the following 't Hooft boundary condition on $\Omega$ :

$$
\begin{aligned}
& \exp \left(\mathrm{i} \xi_{k}\left(x+a^{k}\right)\right) \phi\left(x+a^{k}\right)=\exp \left(\mathrm{i} \xi_{k}(x)\right) \phi(x), \\
& A_{0}\left(x+a^{k}\right)=A_{0}(x), \\
&\left(A_{j}+\partial_{j} \xi_{k}\right)\left(x+a^{k}\right)=\left(A_{j}+\partial_{j} \xi_{k}\right)(x), \\
& x \in \Gamma^{1} \cup \Gamma^{2}-\Gamma^{k}, \quad k=1,2,
\end{aligned}
$$

where $\xi_{1}, \xi_{2}$ are real-valued smooth functions defined in a neighborhood of $\Gamma^{2} \cup$ $\left\{a^{1}+\Gamma^{2}\right\}, \Gamma^{1} \cup\left\{a^{2}+\Gamma^{1}\right\}$, respectively.

It will be more convenient to denote the value of a function $\xi$ at a point $x=$ $s_{1} a^{1}+s_{2} a^{2} \in \bar{\Omega}$ by $\xi\left(s_{1}, s_{2}\right)$. Since $\phi$ is a single-valued complex function, its phase change around $\Omega$ can only be a multiple of $2 \pi$. Thus, the boundary condition (6) leads to the equation

$$
\begin{aligned}
& \xi_{1}\left(1,1^{-}\right)-\xi_{1}\left(1,0^{+}\right)+\xi_{1}\left(0,0^{+}\right)-\xi_{1}\left(0,1^{-}\right) \\
& \quad+\xi_{2}\left(0^{+}, 1\right)-\xi_{2}\left(1^{-}, 1\right)+\xi_{2}\left(1^{-}, 0\right)-\xi_{2}\left(0^{+}, 0\right)+2 \pi N=0,
\end{aligned}
$$

for some integer $N$. As a consequence of (6)-(7), we obtain

$$
\Phi=\int_{\Omega} F_{12} d x=\int_{\partial \Omega} A_{j} d x_{j}=2 \pi N,
$$

which says by (3) that the magnetic flux and electric charge are both quantized in a cell domain. Without loss of generality, we assume $N \geqq 0$ in the sequel.

To calculate the energy, we follow [JW] to rewrite (4) as

$$
\mathscr{E}=\frac{1}{4}\left[\frac{\kappa}{|\phi|} F_{12}+\frac{2}{\kappa}|\phi|\left(|\phi|^{2}-1\right)\right]^{2}+\left|D_{1} \phi+\mathrm{i} D_{2} \phi\right|^{2}+F_{12}+\Lambda
$$

by using the identity $\left|D_{j} \phi\right|^{2}=\left|D_{1} \phi+\mathrm{i} D_{2} \phi\right|^{2}+\mathrm{i}\left[\left(D_{1} \phi\right)\left(D_{2} \phi\right)^{*}-\left(D_{1} \phi\right)^{*}\left(D_{2} \phi\right)\right]$, where

$$
\begin{aligned}
\Lambda & =-F_{12}|\phi|^{2}+\mathrm{i}\left[\left(D_{1} \phi\right)\left(D_{2} \phi\right)^{*}-\left(D_{1} \phi\right)^{*}\left(D_{2} \phi\right)\right] \\
& =\operatorname{Im}\left\{\partial_{j} \varepsilon_{j k} \phi^{*}\left(D_{k} \phi\right)\right\}
\end{aligned}
$$

is a total divergence whose integral over the cell $\Omega$ vanishes by virtue of (6). Thus, applying (8) in the decomposition (9), we find

$$
E(\phi, A)=\int_{\Omega} \mathscr{E} d x \geqq \Phi=2 \pi N,
$$

with equality fulfilled if and only if the pair $(\phi, A)$ satisfies the self-dual equations

$$
\begin{aligned}
D_{1} \phi+\mathrm{i} D_{2} \phi & =0, \\
F_{12}+\frac{2}{\kappa^{2}}|\phi|^{2}\left(|\phi|^{2}-1\right) & =0, \\
\kappa F_{12}+2 A_{0}|\phi|^{2} & =0,
\end{aligned}
$$

subject to the periodic boundary condition (6)-(7). It is straightforward to examine that the solutions of (10) satisfy the full Chern-Simons equations (1). The system (10) was first discovered in the work [HKP, JW] on a full space setting. We have just rederived it on a general periodic lattice cell. The solutions of (10) subject 
to the boundary condition (6) $-(7)$ are condensates which saturate the designated energy level labeled by the integer $N$. Such an energy level is actually determined by the number of vortices confined in the cell. One of the interesting results below is the conclusion that there are only finitely many possible energy levels for each given Chern-Simons coupling parameter $\kappa$.

To formulate our problem properly, recall that the first equation in (10) says that, locally, $\phi$ is holomorphic up to a nonvanishing multiple. Therefore the zeros of $\phi$ are isolated and have integer multiplicities. These zeros give the locations of point vortices. Let the zeros of $\phi$ be $p_{1}, \ldots, p_{m}$ with multiplicities $n_{1}, \ldots, n_{m}$, respectively. Then $N=n_{1}+\cdots+n_{m}$ is the total vortex number which leads to the phase condition (7). Namely, the vortex number gives the winding number of $\phi$ around the boundary of a lattice cell and thus determines the quantized magnetic and electric charges. Counting algebraic multiplicities, an $N$-vortex solution is represented by a solution so that $\phi$ has $N$ zeros. Our basic existence problem for $N$-vortices is: Under what conditions, does the system (10) permit a solution satisfying the periodic boundary condition (6)-(7) and realizing a prescribed $N$-zero set for the Higgs field $\phi$ ? Our main existence theorem is stated as follows.

Theorem 1. Let $p_{1}, \ldots, p_{m} \in \Omega, n_{1}, \ldots, n_{m}$ be some positive integers, and $N=n_{1}+$ $\cdots+n_{m}$. There is a critical value of the coupling parameter, say $\kappa_{c}$, satisfying the upper bound

$$
\kappa_{c} \leqq \frac{1}{2} \sqrt{\frac{|\Omega|}{\pi N}}
$$

so that, for $0<\kappa<\kappa_{c}$, the self-dual Chern-Simons equations (10) subject to the periodic boundary condition (6)-(7) have an $N$-vortex solution $(\phi, A)$, for which, $p_{1}, \ldots, p_{m}$ are the zeros of $\phi$ with multiplicities $n_{1}, \ldots, n_{m}$, respectively, and the energy, magnetic flux, and electric charge are given by the formulas

$$
E=2 \pi N, \quad \Phi=2 \pi N, \quad Q=2 \pi \kappa N .
$$

Moreover, the solution can also be chosen so that the magnitude of $\phi,|\phi|$, has the largest possible values. Such a solution is called a maximal solution which represents a state that is the most superconducting. If $\kappa>\kappa_{c}$, Eqs. (10), subject to (6)-(7) have no solution realizing the zeros $p_{1}, \ldots, p_{m}$, with respective multiplicities $n_{1}, \ldots, n_{m}$.

Furthermore, let the prescribed data be denoted by $S=\left\{p_{1}, \ldots, p_{m}\right.$; $\left.n_{1}, \ldots, n_{m}\right\}$, where the n's may also take zero value, and, write the dependence of $\kappa_{c}$ on $S$ by $\kappa_{c}(S)$. For $S^{\prime}=\left\{p_{1}, \ldots, p_{m} ; n_{1}^{\prime}, \ldots, n_{m}^{\prime}\right\}$, we write $S \leqq S^{\prime}$ if $n_{1} \leqq n_{1}^{\prime}, \ldots, n_{m} \leqq n_{m}^{\prime}$. Then $\kappa_{c}$ is a decreasing function of $S$ in the sense that

$$
\kappa_{c}\left(S^{\prime}\right) \leqq \kappa_{c}(S), \quad \text { whenever } S \leqq S^{\prime},
$$

The inequality (11) says that, for any given coupling parameter $\kappa$, the periodic Chern-Simons system over $\Omega$ can only have finitely many saturated energy levels of the form $E=2 \pi N$.

In the following sections, we present proofs of these results. The construction employing sub/super solutions should be useful to numerical simulations of the multivortex solutions. We will show that the iterations can always start from a largest supersolution (with some point singularities) so that the desired solution obtained in the limit is the maximal solution. Although we do not have accurate 
estimates for the critical number $\kappa_{c}$, the analysis suggests that it seems to depend on the locations of vortices as well as the total vortex number. Another interesting approach is through a variational principle subject to an inequality type constraint. There is a Lagrange multiplier problem if the minimizer occurs at the boundary of the admissible set. A crucial part in the proof is to show that, as far as the parameter $\kappa$ is not too large, the minimizers must be interior. Due to an obstacle arising from the optimal constant in the Trudinger-Moser inequality which is important in our alternative variational approach, we have to assume then that the locations of vortices are sufficiently even in the region. Fortunately, such a gap is filled by the sub/super solution approach.

\section{Construction of Solutions}

For convenience, we introduce the new parameter $\lambda=4 / \kappa^{2}$. In this section, the prescribed zero set of $\phi$ is written $Z(\phi)=\left\{p_{1}, \ldots, p_{N}\right\}$ containing all possible multiplicities. Then the new variable $u=\ln |\phi|^{2}$ reduces the system (10) to the equation

$$
\Delta u=\lambda \mathrm{e}^{u}\left(\mathrm{e}^{u}-1\right)+4 \pi \sum_{j=1}^{N} \delta_{p_{j}} \quad \text { in } \Omega,
$$

where $\delta_{p}$ is the Dirac distribution concentrated at $p \in \Omega$. The boundary condition (6) implies that we are now looking for a solution of (13) defined on the doubly periodic region $\Omega$ or the 2-torus $\Omega=\mathbf{R}^{2} / \Omega$. In the rest of the study, we always observe this assumption.

Conversely, if $u$ is a solution of (13), it is well established that (see [JT]) a solution pair $(\phi, A)$ of (10) may be constructed according to the rules

$$
\begin{aligned}
\phi(z) & =\exp \left(\frac{1}{2} u(z)+\mathrm{i} \sum_{j=1}^{N} \arg \left(z-p_{j}\right)\right), \\
A_{1}(z) & =-\operatorname{Re}\left\{2 \mathrm{i} \partial^{*} \ln \phi(z)\right\} \\
A_{2}(z) & =-\operatorname{Im}\left\{2 \mathrm{i} \partial^{*} \ln \phi(z)\right\}
\end{aligned}
$$

where $z=x_{1}+\mathrm{i} x_{2}$ and $\partial^{*}=\left(\partial_{1}+\mathrm{i} \partial_{2}\right) / 2$. Thus it is enough to solve (13). All the functions below are defined on the 2-torus $\Omega$.

Let $u_{0}$ be a solution of the equation (see [A1])

$$
\Delta u_{0}=-\frac{4 \pi N}{|\Omega|}+4 \pi \sum_{j=1}^{N} \delta_{p_{J}} .
$$

Inserting $u=u_{0}+v$ into (13), we obtain

$$
\Delta v=\lambda \mathrm{e}^{u_{0}+v}\left(\mathrm{e}^{u_{0}+v}-1\right)+\frac{4 \pi N}{|\Omega|} .
$$

Integrating this equation on $\Omega$ yields the constraint

$$
\lambda \int_{\Omega}\left(e^{u_{0}+v}-\frac{1}{2}\right)^{2}=\frac{\lambda}{4}-\frac{4 \pi N}{|\Omega|} .
$$


Thus we are led to the following necessary condition for existence:

$$
\frac{\lambda}{4}>\frac{4 \pi N}{|\Omega|}
$$

as expected. We introduce a monotone iterative scheme to solve (15):

$$
\begin{aligned}
(\Delta-K) v_{n} & =\lambda \mathrm{e}^{u_{0}+v_{n-1}}\left(\mathrm{e}^{u_{0}+v_{n-1}}-1\right)-K v_{n-1}+\frac{4 \pi N}{|\Omega|}, \\
v_{0} & =-u_{0}, \quad n=1,2, \ldots .
\end{aligned}
$$

Lemma 2. Let $\left\{v_{n}\right\}$ be the sequence defined by the scheme (18) with $K \geqq 2 \lambda$. Then

$$
v_{0}>v_{1}>v_{2}>\cdots>v_{n}>\cdots>v_{-}
$$

for any subsolution $v_{-}$of (15). Thus, if there exists a subsolution, the sequence $\left\{v_{n}\right\}$ converges to a solution of (15) in the space $C^{k}(\Omega)$ for any $k \geqq 0$ and such a solution is the maximal solution of the equation.

Proof. We prove (19) by induction.

First, it is standard that $v_{1} \in C^{\infty}\left(\Omega-\left\{p_{1}, \ldots, p_{N}\right\}\right) \cap C^{\alpha}(\Omega) \quad(0<\alpha<1)$. Since $(\Delta-K)\left(v_{1}-v_{0}\right)=0$ in $\Omega-\left\{p_{1}, \ldots, p_{N}\right\}$ (see (14)) and $v_{1}-v_{0}<0$ on $\partial \Omega_{\varepsilon}$, where $\Omega_{\varepsilon}$ is the complement of $\bigcup_{j=1}^{N}\left\{x|| x-p_{j} \mid<\varepsilon\right\}$ in $\Omega$ with $\varepsilon$ sufficiently small, the maximum principle implies $v_{1}-v_{0}<0$ in $\Omega_{\varepsilon}$. Hence $v_{1}-v_{0}<0$ throughout.

Suppose that $v_{0}>v_{1}>\cdots>v_{k}$. We obtain from (19) and $K \geqq 2 \lambda$ that

$$
\begin{aligned}
(\Delta-K)\left(v_{k+1}-v_{k}\right) & =\lambda \mathrm{e}^{2 u_{0}}\left(\mathrm{e}^{2 v_{k}}-\mathrm{e}^{2 v_{k-1}}\right)-K\left(v_{k}-v_{k-1}\right)-\lambda \mathrm{e}^{u_{0}}\left(\mathrm{e}^{v_{k}}-e^{v_{k-1}}\right) \\
& \geqq 2 \lambda \mathrm{e}^{2 u_{0}+2 w}\left(v_{k}-v_{k-1}\right)-K\left(v_{k}-v_{k-1}\right) \\
& \geqq K\left(\mathrm{e}^{2 u_{0}+2 v_{0}}-1\right)\left(v_{k}-v_{k-1}\right)=0
\end{aligned}
$$

where $v_{k} \leqq w \leqq v_{k-1} \leqq v_{0}$. The maximum principle implies $v_{k+1}-v_{k}<0$ in $\Omega$.

Next, we establish the lower bound in (19) in terms of the subsolution $v_{-}$of (15), namely, $v_{-} \in C^{2}(\Omega)$ and

$$
\Delta v_{-} \geqq \lambda \mathrm{e}^{u_{0}+v_{-}}\left(\mathrm{e}^{u_{0}+v_{-}}-1\right)+\frac{4 \pi N}{|\Omega|} .
$$

Initially, we have in view of the definition of $v_{0}$ and (20) that

$$
\begin{aligned}
\Delta\left(v_{-}-v_{0}\right) & =\Delta\left(v_{-}+u_{0}\right) \\
& \geqq \lambda \mathrm{e}^{u_{0}+v_{-}}\left(\mathrm{e}^{u_{0}+v_{-}}-1\right)=\lambda e^{v_{-}-v_{0}}\left(\mathrm{e}^{v_{-}-v_{0}}-1\right)
\end{aligned}
$$

in $\Omega-\left\{p_{1}, \ldots, p_{N}\right\}$. So if $\varepsilon>0$ is small, then $v_{-}-v_{0}<0$ on $\partial \Omega_{\varepsilon}$, and by the maximum principle, we have $v_{-}-v_{0}<0$ in $\Omega_{\varepsilon}$. Hence $v_{-}-v_{0}<0$ throughout $\Omega$.

Now suppose that $v_{-}<v_{k}$ for some $k \geqq 0$. Then (19)-(20) give us

$$
\begin{aligned}
(\Delta-K)\left(v_{-}-v_{k+1}\right) & =\lambda \mathrm{e}^{2 u_{0}}\left(\mathrm{e}^{2 v_{-}}-\mathrm{e}^{2 v_{k}}\right)-K\left(v_{-}-v_{k}\right)-\lambda \mathrm{e}^{u_{0}}\left(\mathrm{e}^{v_{-}}-\mathrm{e}^{v_{k}}\right) \\
& \geqq 2 \lambda \mathrm{e}^{2 u_{0}+w}\left(v_{-}-v_{k}\right)-K\left(v_{-}-v_{k}\right) \\
& \geqq K\left(\mathrm{e}^{2 u_{0}+2 v_{0}}-1\right)\left(v_{-}-v_{k}\right)=0,
\end{aligned}
$$

where $v_{-} \leqq w \leqq v_{k} \leqq v_{0}$. So the maximum principle again implies that $v_{-}<v_{k+1}$. 
The statement of convergence follows from (19) and a standard bootstrap argument.

The proof of the lemma is complete.

Lemma 3. If $\lambda>0$ is sufficiently large, Eq.(15) has a subsolution $v_{-}$satisfying (20).

Proof. Choose small $\varepsilon>0$ so that the balls

$$
B\left(p_{j} ; 2 \varepsilon\right)=\left\{x \in \Omega|| x-p_{j} \mid<2 \varepsilon\right\}, \quad j=1,2, \ldots, N
$$

satisfy $B\left(p_{j} ; 2 \varepsilon\right) \cap B\left(p_{j^{\prime}} ; 2 \varepsilon\right)=\emptyset$ for $p_{j} \neq p_{j^{\prime}}$. Let $f_{\varepsilon}$ be a smooth function so that $0 \leqq f_{\varepsilon} \leqq 1$ and

$$
\begin{aligned}
& f_{\varepsilon}(x)=1, \quad x \in B\left(p_{j} ; \varepsilon\right), \quad j=1,2, \ldots, N, \\
& f_{\varepsilon}(x)=0, \quad x \notin \bigcup_{j=1}^{N} B\left(p_{j} ; 2 \varepsilon\right) .
\end{aligned}
$$

Then we have

$$
C(\varepsilon)=\frac{1}{|\Omega|} \int_{\Omega} \frac{8 \pi N}{|\Omega|} f_{\varepsilon} \leqq \frac{32 \pi^{2} N^{2}}{|\Omega|^{2}} \varepsilon^{2}
$$

Define

$$
g_{\varepsilon}=\frac{8 \pi N}{|\Omega|} f_{\varepsilon}-C(\varepsilon) .
$$

Since $\int g_{\varepsilon}=0$, we know that the equation

$$
\Delta w=g_{\varepsilon}
$$

has a solution which is unique up to an additive constant.

First, from (21), we see that, for $x \in B\left(p_{j} ; \varepsilon\right)$,

$$
g_{\varepsilon} \geqq \frac{4 \pi N}{|\Omega|}\left(2-\frac{8 \pi N}{|\Omega|} \varepsilon^{2}\right)>\frac{4 \pi N}{|\Omega|}
$$

if $\varepsilon$ is small enough. In the following, we fix $\varepsilon$ so that (23) is valid.

Next, we choose a solution of (22), say $w_{0}$, to fulfill

$$
e^{u_{0}+w_{0}}<1, \quad x \in \Omega .
$$

Therefore, for any $\lambda>0$, we have the inequality

$$
\begin{aligned}
\Delta w_{0} & =g_{\varepsilon}>\frac{4 \pi N}{|\Omega|} \\
& \geqq \lambda \mathrm{e}^{u_{0}+w_{0}}\left(\mathrm{e}^{u_{0}+w_{0}}-1\right)+\frac{4 \pi N}{|\Omega|}, \quad x \in B\left(p_{j} ; \varepsilon\right), \quad j=1,2, \ldots, N .
\end{aligned}
$$

Finally, set

$$
\begin{aligned}
& \mu_{0}=\inf \left\{\mathrm{e}^{u_{o}+w_{0}} \mid x \in \Omega-\bigcup_{j=1}^{N} B\left(p_{j} ; \varepsilon\right)\right\}, \\
& \mu_{1}=\sup \left\{\mathrm{e}^{u_{o}+w_{0}} \mid x \in \Omega-\bigcup_{j=1}^{N} B\left(p_{j} ; \varepsilon\right)\right\} .
\end{aligned}
$$


Then $0<\mu_{0}<\mu_{1}<1$ and $\mathrm{e}^{u_{0}+w_{0}}\left(\mathrm{e}^{u_{0}+w_{0}}-1\right) \leqq \mu_{0}\left(\mu_{1}-1\right)=-C_{0}<0$ for $x \in$ $\Omega-\bigcup_{j=1}^{N} B\left(p_{j} ; \varepsilon\right)$. As a consequence, we can choose $\lambda>0$ sufficiently large to fulfill (25) in entire $\Omega$. Thus, $w_{0}$ is a subsolution of (15).

The lemma is proven.

Lemma 4. There is a critical value of $\lambda$, say $\lambda_{c}$, satisfying

$$
\lambda_{c} \geqq \frac{16 \pi N}{|\Omega|},
$$

so that, for $\lambda>\lambda_{c}$, Eq. (15) has a solution; while for $\lambda<\lambda_{c}$, the equation has no solution.

Proof. Suppose that $v$ is a solution of (15). Then $u=u_{0}+v$ verifies (13) and is negative near the point $x=p_{j}, j=1,2, \ldots, N$. Using the maximum principle away from the points $p^{\prime} s$, we find that $u<0$ in $\Omega$.

Define $\Lambda=\{\lambda>0 \mid \lambda$ is such that (15) has a solution $\}$. Then $\Lambda$ is an interval. To show this fact, we prove that, if $\lambda^{\prime} \in \Lambda$, then $\left[\lambda^{\prime}, \infty\right) \subset \Lambda$. In fact, denote by $v^{\prime}$ a solution of (15) at $\lambda=\lambda^{\prime}$. Since $u_{0}+v^{\prime}<0$, we see that $v^{\prime}$ is a subsolution of (15) for any $\lambda \geqq \lambda^{\prime}$. By virtue of Lemma 2 , we obtain $\lambda \in \Lambda$ as desired.

Set $\lambda_{c}=\inf \Lambda$. Then $\lambda>16 \pi N /|\Omega|$ for any $\lambda>\lambda_{c}$ by (17). Taking the limit $\lambda \rightarrow \lambda_{c}$, we arrive at (26). Thus the proof is concluded.

Recall the notation in Theorem 1 for the data of the prescribed zeros of the Higgs field. Namely, $S=\left\{p_{1}, \ldots, p_{m} ; n_{1}, \ldots, n_{m}\right\}, S^{\prime}=\left\{p_{1}, \ldots, p_{m} ; n_{1}^{\prime}, \ldots, n_{m}^{\prime}\right\}$, and the order $S \leqq S^{\prime}$. Then the corresponding statement in Theorem 1 is related to the solvability of the following form of Eq.(13),

$$
\Delta u=\lambda \mathrm{e}^{u}\left(\mathrm{e}^{u}-1\right)+\sum_{j=1}^{m} n_{j} \delta_{p_{j}},
$$

in view of the parameter $\lambda$. We denote the dependence of $\lambda_{c}$ on $S$ by $\lambda_{c}(S)$ (see Lemma 4).

Lemma 5. $\lambda_{c}(S) \leqq \lambda_{c}\left(S^{\prime}\right)$ for $S \leqq S^{\prime}$. Hence (12) holds.

Proof. We need only to show that, if $\lambda>\lambda_{c}\left(S^{\prime}\right)$, then $\lambda \geqq \lambda_{c}(S)$.

Let $u^{\prime}$ be a solution of (27) with $n_{j}=n_{\jmath}^{\prime}, j=1,2, \ldots, m$ and $u_{0}$ satisfy

$$
\Delta u_{0}=-\frac{4 \pi N}{|\Omega|}+4 \pi \sum_{j=1}^{m} n_{j} \delta_{p_{j}}
$$

where $N=n_{1}+\cdots+n_{m}$. Then the substitution $u^{\prime}=u_{0}+v_{-}$leads to

$$
\Delta v_{-}=\lambda \mathrm{e}^{u_{0}+v_{-}}\left(\mathrm{e}^{u_{0}+v_{-}}-1\right)+\frac{4 \pi N}{|\Omega|}+4 \pi \sum_{J=1}^{m}\left(n_{J}^{\prime}-n_{J}\right) \delta_{p_{J}},
$$

which implies in particular that $v_{-}$is a subsolution of (15) in the sense of distributions and (19) holds pointwise. However, since the singularity of $v_{-}$at $x=p_{j}$ is at most of the type $\ln \left|x-p_{1}\right|$, the inequality (19) still results in the convergence of the sequence $\left\{v_{n}\right\}$ to a solution of (15) in any $C^{k}$-norm. In fact, using (19), we see that $\left\{v_{n}\right\}$ converges almost everywhere and is bounded in the $L^{2}$-norm. Hence 
the sequence converges in $L^{2}$. Similarly, the right-hand side of (18) also converges in $L^{2}$. Applying the standard $L^{2}$-estimates we see that the sequence converges in $W^{2,2}$ to a strong solution of (15). Thus a classical solution is obtained. The convergence in $C^{k}$ follows again from a bootstrap argument. This proves $\lambda \geqq \lambda_{c}(S)$. Thus $\lambda_{c}(S) \leqq \lambda_{c}\left(S^{\prime}\right)$ as expected and the lemma is proven.

It is clear that the lemmas of this section furnish the proofs of all the statements made in Theorem 1.

\section{An Alternative Variational Treatment}

In this section we formulate a variational solution of Eq.(15) by using an inequality type constraint. This problem is of independent interest due to the two exponential nonlinear terms in (15). Recall that a similar equation of the form $\Delta v=K_{0}-K^{v}$ arises in the prescribed curvature problem for a 2-surface, compact or noncompact, which has been studied extensively [Av, CY, KW1, KW2, KW3, Mc, Ni]. A basic structure of this latter problem is that it permits a constrained variational principle so that the Lagrange multiplier arising from the constraint naturally recovers the original coefficient in the equation. In our equation (15), the two exponential terms ruin such an approach because the Fréchet derivative of the constraint functional cannot assume a suitable form allowing the recovery of the original equation. Our variational treatment of (15) can be briefly sketched as follows. We first replace the equality constraint (16) by an inequality constraint which is equivalent to the solvability of the equality constraint and defines the admissible set, $\mathscr{A}$, for a suitable objective functional, $I$. We then show that when $\lambda$ is large, the minimizer of $I$ will stay in the interior of $\mathscr{A}$; hence we are able to avoid the Lagrange multiplier problem arising from the equality constraint. Finally we prove that the minimizer obtained in a small space is actually a critical point of $I$ in the usual Sobolev space. Thus a solution of (15) is found.

We concentrate on the case that the points $p_{1}, \ldots, p_{N}$ are so evenly located that the lattice region can be divided into $N$ periodic subregions and that the full problem is an $N$ replication of the problem on a subregion. To solve such a problem, it suffices to consider (15) with $N=1$. This restriction comes from the optimal constant in the Trudinger-Moser inequality which will become clear later.

We use the notation $U=\mathrm{e}^{u_{0}}$. Then (15) takes the form

$$
\Delta v=\lambda U \mathrm{e}^{v}\left(U \mathrm{e}^{v}-1\right)+\frac{4 \pi}{|\Omega|} .
$$

The function $U \geqq 0$ is smooth since it behaves like $\ln |x-p|^{2}$ near the prescribed vortex point $p$.

We shall work on the standard space $H=W^{1,2}(\Omega)$ (the set of doubly periodic functions). Then

$$
H^{\prime}=\left\{\begin{array}{l|l}
v \in H & \int_{\Omega} v=0
\end{array}\right\}
$$

is a closed subspace of $H$ and $H=\mathbf{R}+H^{\prime}$. Namely, for any $v \in H$, there is a unique number $c \in \mathbf{R}$ and $v^{\prime} \in H^{\prime}$ so that

$$
v=c+v^{\prime} \text {. }
$$


Suppose that $v \in H$ given in (29) satisfies (16). Then

$$
\mathrm{e}^{2 c} \int_{\Omega} U^{2} \mathrm{e}^{2 v^{\prime}}-\mathrm{e}^{c} \int_{\Omega} U \mathrm{e}^{v^{\prime}}+\frac{4 \pi}{\lambda}=0
$$

Of course (30) is a quadratic equation in $t=e^{c}$ which has a solution if and only if

$$
\left(\int_{\Omega} U e^{v^{\prime}}\right)^{2}-\frac{16 \pi}{\lambda} \int_{\Omega} U^{2} \mathrm{e}^{2 v^{\prime}} \geqq 0 .
$$

In this case we may choose $c=c\left(v^{\prime}\right)$ in (30) to satisfy

$$
e^{c}=\frac{\int_{\Omega} U e^{v^{\prime}}+\sqrt{\left(\int_{\Omega} U e^{v^{\prime}}\right)^{2}-\frac{16 \pi}{\lambda} \int_{\Omega} U^{2} e^{2 v^{\prime}}}}{2 \int_{\Omega} U^{2} e^{2 v^{\prime}}} .
$$

With $v^{\prime}$ satisfying (31) and $c$ given by (32), we define a functional $I$ on $H^{\prime}$ by the expression

$$
I\left(v^{\prime}\right)=\int_{\Omega}\left\{\frac{1}{2}\left|\nabla v^{\prime}\right|^{2}+\frac{\lambda}{2} U^{2} \mathrm{e}^{2 c+2 v^{\prime}}-\lambda U \mathrm{e}^{c+v^{\prime}}\right\}+4 \pi c .
$$

Set $\mathscr{A}=\left\{v^{\prime} \in H^{\prime} \mid v^{\prime}\right.$ satisfies (31) $\}$. Consider the optimization problem

$$
\min \left\{I\left(v^{\prime}\right) \mid v^{\prime} \in \mathscr{A}\right\} .
$$

We shall find some condition under which the problem (34) has only interior minimizers.

Lemma 6. For $v^{\prime} \in H^{\prime}$ on the boundary of $\mathscr{A}$, namely,

$$
\left(\int_{\Omega} U e^{v^{\prime}}\right)^{2}-\frac{16 \pi}{\lambda} \int_{\Omega} U^{2} e^{2 v^{\prime}}=0,
$$

we have $I\left(v^{\prime}\right) \geqq-4 \pi \ln \lambda-C$ for some constant $C>0$ independent of $\lambda$.

Proof. From (32) and (35), we obtain

$$
e^{c}=\frac{\int_{\Omega} U \mathrm{e}^{v^{\prime}}}{2 \int_{\Omega} U^{2} \mathrm{e}^{2 v^{\prime}}}=\frac{8 \pi}{\lambda \int_{\Omega} U \mathrm{e}^{v^{\prime}}} .
$$

Therefore a simple calculation shows that

$$
I\left(v^{\prime}\right)=-6 \pi+\frac{1}{2}\left\|\nabla v^{\prime}\right\|_{2}^{2}+4 \pi c
$$

where and in the sequel we use \|\|$_{2}$ to denote the usual $L^{2}$-norm on $\Omega$.

We now recall the Trudinger-Moser inequality [A2]

$$
\int_{\Omega} \mathrm{e}^{v^{\prime}} \leqq C(\varepsilon) \exp \left\{\left[\frac{1}{16 \pi}+\varepsilon\right]\left\|\nabla v^{\prime}\right\|_{2}^{2}\right\}, \quad v^{\prime} \in H^{\prime},
$$


where $\varepsilon>0$ is arbitrary. We are going to use (38) to estimate the lower bound of $I$ on $\partial \mathscr{A}$. The optimal constant $1 / 16 \pi$ presents a basic obstacle in the general problem.

We rewrite (36) as

$$
c=\ln 8 \pi-\ln \lambda-\ln \left(\int_{\Omega} U \mathrm{e}^{v^{\prime}}\right) .
$$

Let $p, q>1$ be conjugate exponents to be determined so that $1 / p+1 / q=1$. In view of the Schwartz inequality and (38), we have the following upper bound for $\ln \left(\int U \mathrm{e}^{v^{\prime}}\right)$ :

$$
\begin{aligned}
\ln \left(\int_{\Omega} U \mathrm{e}^{v^{\prime}}\right) & \leqq \frac{1}{p} \ln \left(\int_{\Omega} U^{p}\right)+\frac{1}{q} \ln \left(\int_{\Omega} \mathrm{e}^{q v^{\prime}}\right) \\
& \leqq \frac{1}{p} \ln \left(\int_{\Omega} U^{p}\right)+q\left(\frac{1}{16 \pi}+\varepsilon\right)\left\|\nabla v^{\prime}\right\|_{2}^{2}+\frac{1}{q} \ln C(\varepsilon) .
\end{aligned}
$$

Using (39)-(40) in (37), we arrive at

$$
I\left(v^{\prime}\right) \geqq\left(\frac{1}{2}-4 \pi q\left[\frac{1}{16 \pi}+\varepsilon\right]\right)\left\|\nabla v^{\prime}\right\|_{2}^{2}-4 \pi \ln \lambda-C(\varepsilon, q) .
$$

We can choose suitable $\varepsilon>0$ and $q>1$ above to make the coefficient of the first term on the right-hand side of (41) positive. Thus Lemma 6 is proven.

We now evaluate $I$ at an interior trial point in the admissible set $\mathscr{A}$. For convenience, we choose $v^{\prime}=0$ as a trial element.

Lemma 7. Suppose that $\lambda>0$ is sufficiently large so that

$$
\left(\int_{\Omega} U\right)^{2}-\frac{16 \pi}{\lambda} \int_{\Omega} U^{2}>0
$$

i.e., $v^{\prime}=0$ lies in the interior of $\mathscr{A}$. Then there are constants $C_{1}, C_{2}>0$ independent of $\lambda$ so that $I(0) \leqq-C_{1} \lambda+C_{2}$.

Proof. Assume that $c_{0}=c$ is given by the expression (32) with $v^{\prime}=0$. Equation (30) with $c=c_{0}$ and $v^{\prime}=0$ enable us to obtain

$$
I(0)=-\frac{\lambda}{2} \mathrm{e}^{c_{0}} \int_{\Omega} U+4 \pi\left(c_{0}-\frac{1}{2}\right) .
$$

However, Eq.(32) says that

$$
\frac{\int_{\Omega} U}{2 \int_{\Omega} U^{2}}<\mathrm{e}^{c_{0}}<\frac{\int_{\Omega} U}{\int_{\Omega} U^{2}} .
$$

Inserting this into (42), we obtain

$$
I(0)<-\lambda \frac{\left(\int_{\Omega} U\right)^{2}}{4 \int_{\Omega} U^{2}}+4 \pi \ln \left(\frac{\int_{\Omega} U}{\int_{\Omega} U^{2}}\right) .
$$

Recall that $U$ is independent of $\lambda$. Therefore the lemma follows. 
From the above two lemmas, we see that there is a $\lambda_{0}>0$ so that

$$
I(0)<-1+I\left(w^{\prime}\right), \quad w^{\prime} \in \partial \mathscr{A}, \quad \lambda>\lambda_{0} .
$$

So it is hopeful to get an interior minimizer for (34). From now on we always assume that $\lambda$ is such that (43) holds.

Lemma 8. There are constants $C_{1}, C_{2}>0$ so that

$$
I\left(v^{\prime}\right) \geqq C_{1}\left\|\nabla v^{\prime}\right\|_{2}^{2}-C_{2}, \quad v^{\prime} \in \mathscr{A}
$$

Proof. Using (31)-(32), we have

$$
\mathrm{e}^{c} \geqq \frac{8 \pi}{\lambda}\left(\int_{\Omega} U \mathrm{e}^{v^{\prime}}\right)^{-1} .
$$

As a consequence,

$$
c \geqq-\ln \left(\int_{\Omega} U \mathrm{e}^{v^{\prime}}\right)+\ln \left(\frac{8 \pi}{\lambda}\right) .
$$

On the other hand, the two exponential terms in $I\left(v^{\prime}\right)$ (see (33)) are easily controlled. In fact, using the Schwarz inequality, we have

$$
\int_{\Omega}\left\{\frac{1}{2} U^{2} \mathrm{e}^{2 c+2 v^{\prime}}-U \mathrm{e}^{c+v^{\prime}}\right\} \geqq-2|\Omega| .
$$

Finally, inserting (44)-(45) into (33) and applying the inequality (38) again as in the proof of Lemma 6 , we arrive at the conclusion of the lemma.

Lemma 9. The problem (34) has a minimizer $v^{\prime}$ which lies in the interior of the admissible set $\mathscr{A}$.

Proof. Let $\left\{v_{n}^{\prime}\right\}$ be a minimizing sequence of (34). From Lemma 8 and the Poincaré inequality, we see that $\left\{v_{n}^{\prime}\right\}$ is bounded in $H^{\prime}$. Therefore we may assume without loss of generality that $\left\{v_{n}^{\prime}\right\}$ weakly converges to an element of $H^{\prime}$, say $v^{\prime}$. Since the mapping $H^{\prime} \rightarrow L(\Omega)$ given by $f \mapsto \mathrm{e}^{f}$ is well-defined and compact (see [A1]), we know that $v^{\prime} \in \mathscr{A}$ and $c\left(v_{n}^{\prime}\right) \rightarrow c\left(v^{\prime}\right)$ as $n \rightarrow \infty$. Applying this observation in (33) we see that $v^{\prime}$ is a minimizer of (34). Moreover, (43) implies

$$
I\left(v^{\prime}\right) \leqq-1+\inf \left\{I\left(w^{\prime}\right) \mid w^{\prime} \in \partial \mathscr{A}\right\} .
$$

In other words, $v^{\prime}$ belongs to the interior of $\mathscr{A}$. The lemma is proven.

Since our optimization problem is defined on the subspace $H^{\prime}$ of $H$, it is not obvious whether a critical point of $I$ in $H^{\prime}$ gives rise to a solution of Eq.(28). In the following, we will examine that the composition $c+v^{\prime}$ with $c$ defined by (32) indeed is a critical point of $I$ in the full $H$ and thus solves Eq.(28).

Lemma 10. Let $v^{\prime}$ be the minimizer produced in Lemma 9 and the number $c$ defined by (32). Then $v=c+v^{\prime}$ is a solution of (28). 
Proof. In fact, since $v^{\prime}$ is an interior minimizer, the Fréchet derivative of $I$ at $v^{\prime}$ vanishes. Namely,

$$
\left[\delta I\left(v^{\prime}\right)\right]\left(w^{\prime}\right)=0 \quad \text { for any } w^{\prime} \in H^{\prime}
$$

It is more convenient to rewrite the above equation in the functional form

$$
\begin{aligned}
0= & \int_{\Omega}\left\{\nabla v^{\prime} \cdot \nabla w^{\prime}+\lambda\left[U^{2} \mathrm{e}^{2 c\left(v^{\prime}\right)+2 v^{\prime}}-U \mathrm{e}^{c\left(v^{\prime}\right)+v^{\prime}}\right] w^{\prime}\right\} \\
& +\left[D_{w^{\prime}} c\left(v^{\prime}\right)\right] \int_{\Omega}\left\{\lambda\left[U^{2} \mathrm{e}^{2 c\left(v^{\prime}\right)+2 v^{\prime}}-U \mathrm{e}^{c\left(v^{\prime}\right)+v^{\prime}}\right]+\frac{4 \pi}{|\Omega|}\right\},
\end{aligned}
$$

where the numerical factor in front of the second integral above, i.e.,

$$
D_{w^{\prime}} c\left(v^{\prime}\right)=\left.\frac{d}{d t} c\left(v^{\prime}+t w^{\prime}\right)\right|_{t=0}
$$

is the directional derivative of $c$ at $v^{\prime}$ along $w^{\prime}$. On the other hand, in view of Eq.(30), the second integral above actually vanishes. Thus (46) takes the simplified form

$$
\int_{\Omega}\left\{\nabla v^{\prime} \cdot \nabla w^{\prime}+\lambda\left[U^{2} \mathrm{e}^{2 c+2 v^{\prime}}-U \mathrm{e}^{c+v^{\prime}}\right] w^{\prime}\right\}=0 .
$$

Set $X=L^{2}(\Omega)$ and consider the decomposition $X=\mathbf{R}+X^{\prime}$, where

$$
X^{\prime}=\left\{\begin{array}{l|l}
f \in X & \int_{\Omega} f=0
\end{array}\right\} .
$$

Choose a suitable $\sigma \in \mathbf{R}$ such that

$$
\lambda\left(U^{2} \mathrm{e}^{2 c+2 v^{\prime}}-U \mathrm{e}^{c+v^{\prime}}\right)+\sigma \in X^{\prime} .
$$

Then the relation $H^{\prime} \subset X^{\prime}$ and (47) imply that

$$
\begin{aligned}
0 & =\int_{\Omega}\left\{\nabla v^{\prime} \cdot \nabla \omega^{\prime}+\left(\lambda\left[U^{2} \mathrm{e}^{2 c\left(v^{\prime}\right)+2 v^{\prime}}-U \mathrm{e}^{c\left(v^{\prime}\right)+v^{\prime}}\right]+\sigma\right) w^{\prime}\right\} \\
& =\int_{\Omega}\left\{\nabla v^{\prime} \cdot \nabla\left(a+w^{\prime}\right)+\left(\lambda\left[U^{2} \mathrm{e}^{2 c\left(v^{\prime}\right)+2 v^{\prime}}-U \mathrm{e}^{c\left(v^{\prime}\right)+v^{\prime}}\right]+\sigma\right)\left(a+w^{\prime}\right)\right\}
\end{aligned}
$$

for any $a \in \mathbf{R}$. Consequently,

$$
\int_{\Omega}\left\{\nabla v^{\prime} \cdot \nabla w^{\prime}+\left(\lambda\left[U^{2} \mathrm{e}^{2 c+2 v^{\prime}}-U \mathrm{e}^{c+v^{\prime}}\right]+\sigma\right) w\right\}=0, \quad \forall w \in H .
$$

This equation implies that $v^{\prime}$ is a smooth solution of

$$
\Delta v^{\prime}=\lambda U \mathrm{e}^{c+v^{\prime}}\left(U \mathrm{e}^{c+v^{\prime}}-1\right)+\sigma .
$$

Integrating (48) yields

$$
\lambda \int_{\Omega}\left(U \mathrm{e}^{c+v^{\prime}}-U^{2} \mathrm{e}^{2 c+2 c^{\prime}}\right)=\sigma|\Omega|
$$


Comparing the above equation with (30) we obtain immediately $\sigma|\Omega|=4 \pi$. Thus, by (48), we see that $v=c+v^{\prime}$ solves (28) and the existence proof is complete.

\section{References}

[Ab] Abrikosov, A.A.: On the magnetic properties of superconductors of the second group, Sov. Phys. JETP 5, 1174-1182 (1957)

[A1] Aubin, T.: Nonlinear Analysis on Manifolds: Monge-Ampére Equations. Springer: Berlin Heidelberg, NewYork, 1982

[A2] Aubin, T.: Meilleures constantes dans le théorème d'inclusion de Sobolev et un théorème de Fredholm non linéaire pour la transformation conforme de courburne scalaire, J. Funct. Anal. 32, 148-174 (1979)

[Av] Aviles, P.: Conformal complete metrics with prescribed non-negative Gaussian curvature in $\mathbf{R}^{2}$. Invent. Math. 83, 519-544 (1986)

[CH] Chakravarty, S., Hosotani, Y.: Anyon model on a cylinder. Phys. Rev. D 44, 441-451 (1991)

[CY] Chang, S.Y.A., Yang, P.C.: Prescribing Gaussian curvature on $S^{2}$. Acta Math. 159, 215-259 (1987)

[CHMcY] Chen, X., Hastings, S., McLeod, J.B., Yang, Y.: A nonlinear elliptic equation arising from gauge field theory and cosmology. Proc. R. Soc. London, Series A, 446, 453-478 (1994)

[FM1] Fröhlich, J., Marchetti, P.: Bosonization, topological solitons and fractional charges in two-dimensional quantum field theory. Commun. Math. Phys. 116, 127-173 (1988)

[FM2] Fröhlich, J., Marchetti, P.: Quantum field theories of vortices and anyons. Commun. Math. Phys. 121, 177-223 (1989)

[HKP] Hong, J., Kim, Y., Pac, P.Y.: Multivortex solutions of the Abelian Chern-Simons theory. Phys. Rev. Lett. 64, 2230-2233 (1990)

$[\mathrm{H}]$ Hosotani, Y.: Gauge invariance in Chern-Simons theory on a torus. Phys. Rev. Lett. 62, 2785-2788 (1989)

[IL] Iengo, R., Lechner, K.: Quantum mechanics of anyons on a torus. Nucl. Phys. B 346, 551-575 (1990)

[JLW] Jackiw, R., Lee, K., Weinberg, E.J.: Self-dual Chern-Simons solitions. Phys. Rev. D 42, 3488-3499 (1990)

[JW] Jackiw, R., Weinberg, E.J.: Self-dual Chern-Simons vortices. Phys. Rev. Lett. 64, 2234$2237(1990)$

[JT] Jaffe, A., Taubes, C.H.: Vortices and Monopoles, Boston: Birkhäuser, 1980

[KW1] Kazdan, J.L., Warner, F.W.: Integrability conditions for $\Delta u=k-K e^{2 u}$ with applications to Riemannian geometry. Bull. Am. Math. Soc. 77, 819-823 (1971)

[KW2] Kazdan, J.L., Warner, F.W.: Curvature functions for compact 2-manifolds. Ann. Math. 99, 14-47 (1974)

[KW3] Kazdan, J.L., Warner, F.W.: Curvature functions for open 2-manifold. Ann. Math. 99, 203-219 (1974)

[Mc] McOwen, R.C.: Conformal metrics in $\mathbf{R}^{2}$ with prescribed Gaussian curvature and positive total curvature. Indiana U. Math. J. 34, 97-104 (1985)

[Ni] Ni, W.-M.: On the elliptic equation $\Delta u+K(x) \mathrm{e}^{2 u}=0$ and conformal metrics with prescribed Gaussian curvature. Invent. Math. 66, 343-352 (1982)

[O] Olesen, P.: Solition condensation in some self-dual Chern-Simons theories. Phys. Lett. B 265, 361-365 (1991); Erratum, 267, 541 (1991)

[P] Polychronakos, A.: Abelian Chern-Simons theories in 2+1 dimensions. Ann. Phys. 203, 231-254 (1990)

[RSS] Randjbar, S., Salam, A., Strathdee, J.A.: Anyons and Chern-Simons theory on compact spaces of finite genus. Phys. Lett. B 240, 121-126 (1990)

['t H] Hooft, G. 't.: A property of electric and magnetic flux in nonabelian gauge theories. Nucl. Phys. B 153, 141-160 (1979) 
[SY1] Spruck, J., Yang, Y.: The existence of non-topological solitons in the self-dual ChernSimons theory. Commun. Math. Phys. 149, 361-376 (1992)

[SY2] Spruck, J., Yang, Y.: Topological solutions in the self-dual Chern-Simons theory: Existence and approximation. Ann. l'I. H. P.- Anal. non Linéaire, to appear

[T1] Taubes, C.H.: Arbitrary $N$-vortex solutions to the first order Ginzburg-Landau equations. Commun. Math. Phys. 72, 277-292 (1980)

[T2] Taubes, C.H.: On the equivalence of the first and second order equations for gauge theories. Commun. Math. Phys. 75, 207-227 (1980)

[WY] Wang, S., Yang, Y.: Abrikosov's vortices in the critical coupling. SIAM J. Math. Anal. 23, 1125-1140 (1992)

Communicated by T. Spencer 